\title{
Simulation model of selective assembly of two parts with sorting by the estimated values
}

\author{
Oleg Filipovich ${ }^{1, *}$, and Vadim Kopp ${ }^{1}$ \\ ${ }^{1}$ Sevastopol State University, 299053, Sevastopol, Russian Federation
}

\begin{abstract}
The process of one-parameter selective assembly of two parts is considered. Methods for reducing the probability of sorting errors are given. It is suggested to perform the sorting process according to the values of the parameter estimates. For this estimate, a recursion algorithm (the Kalman filter) is used in each cycle. In the environment of GPSS World, a simulation model of selective acquisition and assembly of two parts for determining the parameters of the assembly process is constructed. Comparative modeling results are presented that prove the effectiveness of the algorithm for estimating parameters for selective assembly. The variants of using the algorithm and the prospects for further research are suggested.
\end{abstract}

\section{Introduction}

The process of one-parameter selective assembly of two types of parts by parameters $x_{i}$ ( $i=1,2$ ), manufactured by $N$ - batch of parts, is considered. This process is conditionally characterized by the scheme shown in Figure 1, the notation on which corresponds to those adopted in [1].

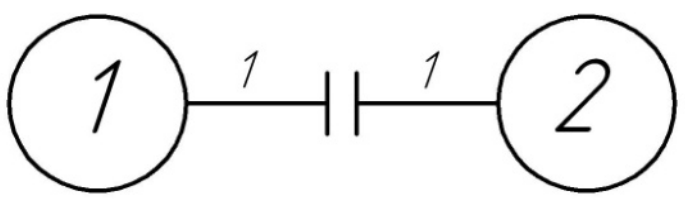

Fig. 1. Selection scheme

In this case, the parameters are the linear dimensions of the mating surfaces or the deviations of these dimensions from their nominal values. One of the main indicators of the quality of assembly is the accuracy of the output parameter of the product (joint) associated with the known dependence $x_{i}$.

The main stages of selective assembly of engineering products are discussed in detail in [1-4]. The key step is the sorting of the parts into size groups, which is provided in the

\footnotetext{
* Corresponding author: ophis1@yandex.ru
} 
production line by control and sorting machines. The control of the details is accompanied by random errors, the appearance of which leads to the fact that instead of the true value of the parameter $x_{k, i}$ in the measurement process, a value $z_{k, i}$ usually associated with the initial dependence becomes known:

$$
z_{k, i}=x_{k, i}+\delta_{k, i}
$$

where $\delta_{k, i}$ - random component of the measurement error of the $i$-th parameter, $k$ - the cycle number corresponding to the serial number of the part in the batch $(k=\overline{1, N})$.

As a result of sorting the parts according to the conventional true values of the parameter $z$, sorting errors can occur: the hit of a part in one of the neighboring selective groups instead of the required group, wherever it would get when sorting by the true values at $\delta_{k, i} \rightarrow 0$. In turn, sorting errors will affect the quality of products: using a one-variant selection algorithm (selection from the same selective groups), products obtained from such parts will be knowingly defective in the output. Obviously, the probability of obtaining such events will be less, the smaller the magnitude of the measurement error $\delta_{k, i}$, i.e. more proximity of the actual value of the parameter to the true value.

Reduce this probability in several ways:

1) by choosing the accuracy of the means of measuring technology, which provides the measurement error required for the given criteria;

2) the use of methods for the optimal evaluation of the parameters of parts in the presence of a priori information.

Here it is proposed to implement the second method by using as a criterion the minimum root-mean-square value of the estimation error. A significant contribution to the creation of an effective procedure for calculating the state estimation was introduced as a result of the development of the Kalman algorithm and its subsequent modifications [5-12]. The application of this algorithm for the control of technological equipment is described in $[13,14]$.

The purpose of this work is to modify the simulation model of selective assembly of two parts by implementing the sorting algorithm based on the values of the parameters obtained as a result of their recurrent evaluation.

\section{Simulation model}

Simulation models are widely used to analyze the behavior of stochastic dynamic systems consisting of a large number of interacting elements and processes. The work [15] is devoted to the construction of the basic model of the process of a one-parameter selective assembly. The model is built in the GPSS World - the modern version of the General Purpose Simulation System [16-18] and consists of three main segments.

1. Set the initial data: the number of sorting groups, batch sizes, the capacity of the assembly storage, the data for determining the value of the output parameter of the assembled joint and the parameters of the assembly process, data about the mated parameters of the product, the limiting values of the measurement errors. In addition, the tables necessary to collect statistics are described here.

2. Simulation of the measurement and sorting process. The implementation of each individual part in the model is its dynamic object - transact. Generates a series of transactions, while the size of the series is determined by the size of the batch. Each transaction in the parameters is sequentially recorded with true and valid values, as well as 
with the number of the selective group determined by the special procedure. The procedure is organized with the help of subroutines executed in the PLUS programming language, built in GPSS World. Transactions, divided in this way into groups, fall into the user lists with certain numbers.

3. Simulation of the assembly process using the one-variant selection algorithm. This segment of the model works in parallel with the second one. Generates an output transaction, which is delayed in a single-channel device that simulates the search period of the set. If the set can be assembled from the current group numbers and there are the necessary number of transactions in the corresponding user lists, then they are output from the lists and are delivered to the assembly storage. The procedure is repeated until the last assembly of the same selective groups is assembled.

After running the model, the report presents the data necessary for further analysis:

1) information on statistical distribution densities, on average values and standard deviations in the sizes of parts in the batch (true and actual), output index, measurement errors;

2) the number of transactions remaining in the user lists, which constitute work in progress and preliminary scrappage;

3 ) the total number of assembled sets and accepted assembled sets.

The research of the influence of measurement accuracy on the parameters of the assembly process using simulation models are devoted the works $[19,20]$. They consider the process of assembling a joint consisting of two parts that form a fit connection with clearance. For selection, the following rule was adopted: the dimensions of the parts selected in a certain way from the corresponding groups, during assembly should provide a clearance $S$ lying within

$$
S_{\min } \leq S \leq S_{\max }
$$

while the details of these groups should be completely interchangeable. To determine the number of the selective group, use the conventional true value of the parameter, calculated from formula (1).

We modify the given simulation model, in particular, its second segment. Consider the process of forming parts on metal cutting machines, which under real conditions is performed under the influence of many random disturbances. Taking into account the dynamics of the $x_{i}$ parameter change and its random fluctuations, we can write:

$$
x_{k, i}=x_{k-1, i}+l_{k-1, i}+\xi_{k-1, i}+u_{k-1, i}, i=1,2,
$$

where $x_{k, i}$ and $x_{k-1, i}$ are the true values of the parameters on the $k$-th and (k-1)-th cycles of the process, respectively; $l_{k-1, i}$ - a known quantity that determines the evolution of the system; $\xi_{k-1, i}$ - instantaneous handling error; $u_{k-1, i}$ - control action to the machine tool.

We assume the following.

1. The value $l_{k}$ is the offset of the tuning level, caused by the wear of the cutting tool, determined by formula

$$
l_{k}=\alpha k,(k=\overline{1, N})
$$

where $\alpha$ - the wear rate of the tool per cycle.

2. The initial setting of the machines is carried out absolutely exactly on the coordinates of the middle of the extended tolerance interval of a part of each type. 
3. The control action $u_{k, i}$ is a deterministic quantity, which is proposed for each processing cycle as the value of the estimate computed in the previous cycle and taken with the opposite sign.

Equations (1) and (4) form discrete scalar systems that describe the dynamical process under study in the state space. Suppose also that all random variables are independent and have zero mathematical expectations. To solve the problem of estimating the parameters of parts, we apply the recursive algorithm. The Kalman filter equation for such a system has the form

$$
\begin{aligned}
& \hat{x}_{k, i}=\hat{x}_{k-1, i}+\alpha+\xi_{k-1, i}+u_{k-1, i}+K_{k, i}\left(z_{k, i}-\hat{x}_{k-1, i}-\alpha-u_{k-1, i}\right), \\
& K_{k, i}=\frac{V_{\varepsilon k-1, i}+\sigma_{\xi, i}^{2}}{V_{\varepsilon k-1, i}+\sigma_{\xi, i}^{2}+\sigma_{\delta, i}^{2}}, V_{\varepsilon k, i}=M\left[\varepsilon_{k, i}^{2}\right]=\frac{\sigma_{\delta, i}^{2}\left(V_{\varepsilon k-1, i}+\sigma_{\xi, i}^{2}\right)}{V_{\varepsilon k-1, i}+\sigma_{\xi, i}^{2}+\sigma_{\delta, i}^{2}},
\end{aligned}
$$

where $\hat{x}_{k, i}, \hat{x}_{k-1, i}$ - estimates of $x_{k, i}$ and $x_{k-1, i} ; K_{k, i}$ - Kalman filter gain; $M$ - the symbol of mathematical expectation; $\varepsilon_{k, i}$ - estimation error, $\varepsilon_{k, i}=x_{k, i}-\hat{x}_{k, i} ; \sigma_{\xi}^{2}$ and $\sigma_{\delta}^{2}-$ variances of quantities $\xi$ and $\delta$.

In the modified model, the sorting process is performed not according to conventional true $z_{k, i}$, but by parameter of estimates $\hat{x}_{k, i}$. Further, the values of the output parameters are calculated and the condition (2) is verified. If it is executed, then the sorting according to the parameter estimates is carried out correctly and the set is accepted, if not - defective. Assembly set is made in any case.

\section{Simulation example}

Here is an example of modeling the process of selective assembly using the constructed model. Suppose that batches of parts of identical volumes $N=1000$ are produced. Let's assume that

1) the random variable $\xi$ has a normal distribution with zero mathematical expectation and standard deviations $\sigma_{\xi 1}=0,0028 \mathrm{~mm} ; \sigma_{\xi 2}=0,0024 \mathrm{~mm}$.

2) The random variable $\delta$ is determined on the interval $\left[-\Delta_{i}, \Delta_{i}\right]\left(\Delta_{i}-\right.$ the limiting value of the measurement error of the $i$-th part, $\Delta_{1}=\Delta_{2}=0,0015 \mathrm{~mm}$ ), has a truncated normal distribution with zero mathematical expectation and standard deviation $\sigma_{\delta i}=\frac{\Delta_{i}}{2}$;

3) The values of the extended tolerances for the parts of both types are taken equal to each other $T_{1}=T_{2}=0,018 \mathrm{~mm}$; the number of selective groups of accepted parts is fixed, $L_{1}=L_{2}=6$; intervals of extended tolerances $(\mathrm{mm}) \quad[-0,018 ; 0]$ and $[0 ; 0,018]$ respectively, for parts of the first and second types; the selective tolerances are the same, the output (mm) $0,015 \leq S \leq 0,021$.

The results of running the initial and modified models using fifty different random number generators for the given initial data:

- the average number of accepted assembled sets for sorting according to the conventional true values $z_{k, i}$ is 752 ; 
- the average number of accepted assembled sets for sorting by parameter estimates $\hat{x}_{k, i}$ is 765 ;

- the average number of accepted assembled sets for sorting by true parameter values $x_{k, i}$ is 786 .

\section{Conclusion}

These results indicate the effectiveness of the application of the parameter estimation algorithm for selective assembly, used here as one of the ways to reduce the probabilities of sorting errors. In the future, the algorithm is proposed to be used to estimate the probabilistic indicators of products batches selectively collected among themselves.

The reported study was funded by RFBR and Government of the Sevastopol according to the research project №18-48-920014.

\section{References}

1. V.Y. Katkovnik, A.I. . Savchenko. Fundamentals of the theory of selective assembly (Polytechnics, Leningrad, 1991).

2. M.A. Bonch-Osmolovsky. Selective assembly (Mechanical Engineering, Moscow, 1974).

3. P.I. Bulovsky, G.V. Krylov, V.A. Lopukhin. Automation of selective assembling of instruments (Mechanical Engineering, Leningrad, 1978).

4. S.M. Kannan and V. Jayabalan. International Journal of Production Research, 39/9, 1851-1863 (2001).

5. J.C. Spall. Introduction to Stochastic Search and Optimization: Estimation, Simulation, and Control (Hoboken, New Jersey: Wiley, 2003).

6. G. Welch, G. Bishop. An Introduction to the Kalman Filter (Los Angeles, 2001).

7. B.D.O. Anderson, J.B. Moore. Optimal Filtering (New York: Dover, 2005).

8. Yu.E. Voskoboinikov. Recursive estimation of the state vector of the dynamic system (Novosibirsk: NSTU, 2014).

9. A. Gelb. Applied Optimal Estimation (MIT Press, Cambridge, MA., 1974)

10. Mohinder S. Grewal, P. Andrews Angus. Kalman Filtering: Theory and Practice (Upper Saddle River, NJ USA, Prentice Hall, 1993).

11. O.L.R. Jacobs. Introduction to Control Theory, 2nd Edition (Oxford University Press, 1993).

12. I.N. Sinitsyn. Kalman and Pugachev Filters (Moscow, 2006).

13. O.V. Filipovich, V.Y. Kopp, M.I. Garmatyuk. Assembling in Mechanical Engineering and Instrument-Making, 5 (142), 3-7 (2012).

14. V.D. Boev. Modeling of systems, tools of GPSS World (BHV-Petersburg, St.Petersburg, 2004).

15. E.M. Kudryavtsev. GPSS World. Fundamentals of simulation of various systems (DMK Press, Moscow, 2004).

16. R.F. Malikov. Workshop on discrete-event modeling of complex systems in the advanced editor GPSS World (BSPU, Ufa, 2017). 
17. O. Filipovich, V. Kopp, N. Voloshina, A. Bokhonsky. MATEC Web of Conferences, 129 (2017), 03026 (2017).

18. O.V. Filipovich. Fundamental and Applied Problems of Engineering and Technology 4-2 (324), 134-140 (2017).

19. O.V. Filipovich, G.V. Nevar. Automation and Instrumentation: Problems, Solutions: Proceedings of the International Scientific and Technical Conference, 99 (2017).

20. O.V. Filipovich, G.V. Nevar. Izvestiya TulGU, 9-1, 428-437 (2017). 